\title{
Fat Tail Model for Simulating Test Systems in Multiperiod Unit Commitment
}

\author{
J. A. Marmolejo ${ }^{1}$ and R. Rodriguez ${ }^{2}$ \\ ${ }^{1}$ Faculty of Engineering, Anahuac University, 52786 Mexico City, MEX, Mexico \\ ${ }^{2}$ School of Economics, National Polytechnic Institute, 11350 Mexico City, MEX, Mexico \\ Correspondence should be addressed to J. A. Marmolejo; jose.marmolejo@anahuac.mx
}

Received 18 September 2014; Accepted 7 January 2015

Academic Editor: Wei-Chiang Hong

Copyright ( 2015 J. A. Marmolejo and R. Rodriguez. This is an open access article distributed under the Creative Commons Attribution License, which permits unrestricted use, distribution, and reproduction in any medium, provided the original work is properly cited.

\begin{abstract}
This paper describes the use of Chambers-Mallows-Stuck method for simulating stable random variables in the generation of test systems for economic analysis in power systems. A study that focused on generating test electrical systems through fat tail model for unit commitment problem in electrical power systems is presented. Usually, the instances of test systems in Unit Commitment are generated using normal distribution, but in this work, simulations data are based on a new method. For simulating, we used three original systems to obtain the demand behavior and thermal production costs. The estimation of stable parameters for the simulation of stable random variables was based on three generally accepted methods: (a) regression, (b) quantiles, and (c) maximum likelihood, choosing one that has the best fit of the tails of the distribution. Numerical results illustrate the applicability of the proposed method by solving several unit commitment problems.
\end{abstract}

\section{Introduction}

Unit commitment, economic dispatch, and other optimization problems for electrical power systems have been studied for more than five decades. A definition of economic dispatch is the operation of generation facilities to produce energy at the lowest cost to reliably serve consumers, recognizing any operational limits of generation and transmission facilities.

In the typical Unit Commitment [1] the problem is determine the mix of generators and their estimated output level to meet the expected demand of electricity over a given time horizon (a day or a week), while satisfying the load demand, spinning reserve requirement, and transmission network constraints. An electric network consists of many generation nodes with various generating capacities and cost functions, lines of transmission, and nodes of power demand.

The application of optimization models for electrical power systems is marked by constant development for new algorithms like exact methods, metaheuristics, and hybrid strategies. However, to benchmark the performance and solution quality for any solution technique, it is necessary to have a variety of electrical test systems.
Nowadays, we still lack the existence of standardized test systems that can be used to benchmark the performance and solution quality of proposed techniques. Many papers consider different test systems, which make it very difficult to perform a proper comparison between the different methods that have been proposed [2].

In [3], they refer that the existing IEEE test systems developed are mainly used for reliability, power flow, and stability analysis but not for economic analysis. A short time ago, some panels focused on the development of standard test systems of transmission and distribution systems for economic analysis have emerged. In 2007 the IEEE Working group (WG) on Test Systems for Economic Analysis was created, sponsored by IEEE System Economics subcommittee [3].

In this sense, this work proposes a way to generate test cases that can be used to unit commitment based on the use of Chambers-Mallows-Stuck method for simulating stable random variables. The stable distributions theory was first developed in the 20s of last century by Lévy [4]. Since then, this distribution has been applied in different areas of knowledge, such as economics, physics, engineering, and hydrology. The reason is that some phenomena of nature, 
like electrical demand, cannot be described assuming normal distribution as they present observations with extreme values, which characterize the instability of the series and denote the presence of heavy tails, an effect known as impulsivity.

Usually electrical demand presents a greater degree of impulsivity that the normal distribution cannot describe due to the presence of peaks in the series during the hours of the day and seasons of high-energy demand.

For this, we use Chambers-Mallows and Stuck algorithm for simulating alpha stable random variables characterizing demand patterns of real electrical systems. The use of Chambers-Mallows-Stuck method for simulating stable random variables provides a new way to generate test systems widely used in power systems research. Modeling the demand through the use of alpha stable distribution can catch the real behavior of the electrical demand and build possible extreme scenarios, and each scenario corresponds to a priceelastic demand curve. The simulations are based on real observations of demand for different reliability test systems. Electrical network data are taken from the 24 and 118 bus IEEE test systems $[5,6]$ and a portion of electric energy system of Mainland Spain [7]. Cost functions of the thermal plants data are taken from the literature.

After the elaboration of these test cases, we tested a mixed integer nonlinear formulation of unit commitment problem based on these cases, in order to obtain estimation about the performance of these new test systems.

\section{Stable Distributions}

The stable distributions theory was first developed in the 20s of last century by Lévy [4]. Since then, this distribution has been applied in different areas of knowledge, such as economics, physics, hydrology, and signal processing. However, it was not until the work of Mandelbrot in 1960s that the $\alpha$ stable distributions were popularized. Mandelbrot proposed a revolutionary theory based on this distribution to solve the problem of price fluctuations, later shown that many other economic variables follow a $\alpha$-stable distribution.

Significantly, the $\alpha$-stable distribution meets the central limit theorem and the stability property (which denotes that stable distributions are isomorphic) that also contains the normal distribution as a special case of this. On the other hand, there is some complexity in working with such distributions, since they lack, in general, of an analytical expression. Although given recent computational advances, it is now possible to apply them with more feasibility to different areas.

Definition 1. A random variable $X$ has $\alpha$-stable distribution having the following characteristic function [8]:

$$
\begin{array}{r}
\varphi(w) \\
=\left\{\begin{array}{r}
\exp \left\{-|\gamma w|^{\alpha}\left[1-i \operatorname{sign}(w) \beta \tan \left(\frac{\pi \alpha}{2}\right)\right]+i \delta w\right\}, \\
\exp \left\{-|\gamma w|\left[1+i \operatorname{sign}(w) \beta \frac{2}{\pi} \log (|w|)\right]\right. \\
+i \delta w\}, \\
(\alpha=1),
\end{array}\right.
\end{array}
$$

where $\operatorname{sign}(w)=w /|w|$ and $\alpha \in(0,2]$, whose parameters are defined as follows: $\alpha$ represents the characteristic exponent, which controls the degree of impulsiveness of the random variable $W$. Moreover, the parameter $\beta \in[-1,+1]$ controls the symmetry of distribution $(\beta=0, \alpha$-stable distribution symmetrical, $\beta=1$, and $\beta=-1$ to the family of $\alpha$-stable distributions positive and negative, resp.). While $\gamma>0$ is a scale parameter, also called dispersion, and $\delta$ is the position parameter.

Remarkably, if the expression of the characteristic function parameter, $\alpha=2$, then the parameter $\beta$ becomes meaningless, since $\beta \tan \pi=0$. In this case, the characteristic function becomes

$$
\varphi(w)=\exp \left\{-|\gamma w|^{2}+i \delta w\right\}
$$

The above expression is the characteristic function of a Gaussian random variable with mean $\delta$ and variance $\sigma^{2}=2 \gamma^{2}$. So, from the definition above, we also can show that the normal distribution is a particular case of $\alpha$-stable distribution. Given the properties of $\alpha$-stable distribution above, it follows that its use is justified in the same way as the Gaussian distributions and not only that, but the Gaussian distribution is a particular case of stable and therefore the range of application of $\alpha$-stable distributions is even wider than the normal distribution.

This is mainly due to the existence and continuity of the probability density function of $\alpha$-stable, but with a few exceptions, it cannot be expressed in a compact way. In other words, the integral with respect to $(w)$ of the characteristic function (1) only has an analytical solution for the described cases, denoting the $\alpha$-stable distribution by four parameters $f_{\alpha, \beta(\cdot \mid \gamma, \delta)},[8]$.

A $\alpha$-stable distribution is with the following parameters.

(i) $f_{2,0}(\cdot \mid \gamma, \delta)$ is a Gaussian distribution with mean $\delta$ and variance $2 \gamma^{2}$ :

$$
f_{2,0}(x \gamma, \delta)=N\left(x \delta, 2 \gamma^{2}\right)=\frac{1}{2 \gamma \sqrt{\pi}} \exp \left\{\frac{(x-\delta)^{2}}{4 \gamma^{2}}\right\},
$$

(ii) $f_{1,0}(\cdot \mid \gamma, \delta)$ is a Cauchy distribution with density:

$$
f_{1,0}(x \gamma, \delta)=p \text { Cauchy }(x \mid \delta, \gamma)=\frac{\gamma}{\pi\left((x-\delta)^{2}+\gamma^{2}\right)},
$$

(iii) $f_{1 / 2,1}(\cdot \mid \gamma, \delta)$ is a Levy distribution with density:

$$
\begin{aligned}
f_{1 / 2,1}(x \mid \gamma, \delta) & =p \operatorname{Lévy}(x \mid \delta, \gamma) \\
& =\left(\frac{\gamma}{2 \pi}\right)^{1 / 2} \frac{1}{(x-\delta)^{3 / 2}} \exp \left\{-\frac{\gamma}{2(x-\delta)}\right\} .
\end{aligned}
$$

The current computational developments and their power applied to the distribution parameter calculations of $\alpha$ stable distributions had been a key elementin the recent use of 
such distributions in many areas. The statistical significance of the estimated parameters can be contrasted by different statistical tests, and the hypothesis is as follows.

H0: the series is distributed as $\alpha$-stable distribution.

H1: the series is not distributed as $\alpha$-stable distribution.

The Anderson-Darling test showed greater efficiency in the case of heavy tailed series, its relevance, and use has been generally accepted for the analysis of stable series [9].

2.1. Stable Random Variable. For a random sample with $\alpha$-stable distributions the Chambers-Mallows-Stuck [10] method. A random variable $X$ with distribution $f_{\alpha, \beta}(X \mid$ $1,0)$ can be generated from a nonlinear transformation of two random variables independent, one uniform $(V)$ and another exponential $(W)$ using the following theorem.

Theorem 2. Let $(V)$ be a uniform random variable in the interval $(-\pi / 2, \pi / 2)$ and let $(W)$ be an exponential random variable with mean equal one. If $V$ and $W$ are independent, then

$$
\begin{aligned}
X= & S_{\alpha, \beta} \frac{\sin \left(\alpha\left(V+B_{\alpha, \beta}\right)\right)}{(\cos V)^{1 / \alpha}} \\
& \cdot\left(\frac{\cos \left((1-\alpha) V+B_{\alpha, \beta} \alpha\right)}{W}\right)^{(1-\alpha) / \alpha},
\end{aligned}
$$

where $X$ follows a stable distribution with $f_{\alpha, \beta}(X \mid 1,0)$, where

$$
\begin{aligned}
B_{\alpha, \beta} & =\frac{\arctan (\beta \tan (\pi \alpha / 2))}{\alpha}, \\
S_{\alpha, \beta} & =\left(1+\beta^{2} \tan ^{2} \frac{\pi \alpha}{2}\right)^{1 / 2 \alpha} .
\end{aligned}
$$

Once you get the variable $(X)$ is generated a variable with stable distribution for any value of the parameters $\alpha, \beta, \sigma y \mu$. If $X \sim f_{\alpha, \beta}(X \mid 1,0)$, then

$$
\begin{gathered}
f_{\alpha, \beta}(\gamma, \mu) \sim \gamma X+\mu \quad \text { if } \alpha \neq 1, \\
f_{\alpha, \beta}(\gamma, \mu) \sim \gamma X+\frac{2}{\pi} \beta \gamma \ln \gamma+\mu \quad \text { if } \alpha=1 .
\end{gathered}
$$

\section{The Multiperiod Unit Commitment Model}

In this work we address a multiperiod unit commitment based on [11] notation, where network constraints are represented through a DC model [7]. The following notation is used in the mathematical model.

\section{Constants Are as Follows}

$A_{j}$ : start-up cost of power plant $j$,

$B_{n m}$ : susceptance of line $n-m$,

$C_{n m}$ : transmission capacity limit of line $n-m$,

$D_{n k}$ : load demand at node $n$ during period $k$,
$E_{j}\left(t_{j k}\right)$ : nonlinear function representing the operating cost of power plant $j$ as a function of its power output in period $k$,

$E_{j 1}$ : linear coefficient of operating cost for the plant $j$,

$E_{j 2}$ : quadratic coefficient of operating cost for the plant $j$,

$F_{j}$ : fixed cost of power plant $j$,

$K_{n m}$ : conductance of line $n-m$,

$R_{k}$ : spinning reserve requirement during period $k$,

$\overline{T_{j}}$ : maximum power output of plant $j$,

$T_{j}$ : minimum power output of plant $j$,

$n r$ : reference node with angle cero.

\section{Variables Are as Follows}

$t_{j k}$ : power output of plant $j$ in period $k$,

$v_{j k}$ : binary variable which is equal to 1 when plant $j$ is committed in period $k$

$y_{j k}$ : binary variable which is equal to 1 when plant $j$ is started up at the beginning of period $k$,

$\delta_{n k}$ : angle of node $n$ in period $k$.

Sets Are as Follows

$J$ : set of indices of all plants,

$K$ : set of period indices,

$N$ : set of indices of all nodes,

$\Lambda_{n}$ : set of indices of the power plants $j$ at node $n$,

$\Omega_{n}$ : set of indices of nodes connected and adjacent to node $n$.

The objective is minimizing a function that includes fixed cost, start-up cost, and operating cost. A second order polynomial describes the variable costs as a function of the electric power:

$$
\operatorname{Min} Z=\sum_{k \in K} \sum_{j=J}\left[F_{j} v_{j k}+A_{j} y_{j k}+E_{j}\left(t_{j k}\right)\right] .
$$

There is a power balance constraint per node and time period. In each period, the production has to satisfy the demand and losses in each node. Line losses are modeled through cosine approximation and it is assumed that the demand for electric energy is known and is discretized into $t$ periods:

$$
\begin{aligned}
& \sum_{j \in \Lambda_{n}} t_{j k}+\sum_{m \in \Omega_{n}} B_{n m}\left[\delta_{m k}-\delta_{n k}\right] \\
& \quad-\sum_{m \in \Omega_{n}} K_{n m}\left[1-\cos \left(\delta_{m k}-\delta_{n k}\right)\right]=D_{n k} \quad \forall n \in N, \forall k \in K .
\end{aligned}
$$

Spinning reserve requirements are modeled. In each period the running units have to be able to satisfy the demand and the prespecified spinning reserve:

$$
\sum_{j \in J} \overline{T_{j}} v_{j k} \geq \sum_{n \in N} D_{n k}+R_{k} \quad \forall k \in K .
$$


Each unit has a technical lower and upper bound for the power production:

$$
\underline{T_{j}} v_{j k} \leq t_{j k} \leq \overline{T_{j}} v_{j k} \quad \forall j \in J, \forall k \in K
$$

Transmission capacity limits of lines avoid dynamic stability system problems:

$$
-C_{n m} \leq B_{n m}\left[\delta_{m k}-\delta_{n k}\right] \leq C_{n m} \quad \forall n \in N, \forall k K, \forall m \in \Omega_{n} .
$$

This constraint holds the logic of running, start-up, and shut-down of the units. A running unit cannot be started up:

$$
y_{j k} \geq v_{j k}-v_{j k-1} \quad \forall j \in J, \forall k .
$$

Angle in all buses has a lower and upper bound:

$$
-\pi \leq \delta_{n k} \leq \pi \quad \forall n \in \frac{N}{\{n r\}}, \forall k \in K .
$$

\section{Test Systems Generation}

To generate new test systems (instances) by the methodology proposed we worked with three standardized test systems:

(i) System I: based on 104-bus electric energy system of Mainland Spain with 104 nodes, 62 thermal units, and 160 transmission lines [7],

(ii) System II: based on IEEE-24 bus test system with 24 nodes, 24 thermal units, and 38 transmission lines [5],

(iii) System III: based on IEEE-118 bus test system with 118 nodes, 54 thermal units, and 186 transmission lines [6].

All instances consider a 24-hour planning horizon with one period per hour. We use stable distribution to model the demand from the original systems. After a quantile analysis, we conclude that the three systems do not follow a normal distribution and show the presence of extreme values and fat tails (see Figures 1, 2, and 3).

Normal test shows that three systems do not follow a normal distribution and for the nature of the series have a fat tails characteristics (See Tables 1, 2, and 3).

The data do not follow a normal distribution and have fat tails, because we use alpha stable distribution to fit the data. It was felt that $S_{1}$ parameterization is usually used [12] for modeling stable data:

$$
\begin{aligned}
& \varphi(k)= \ln f_{c}(k) \\
&=\left\{\begin{array}{l}
i \delta k-\gamma^{\alpha}|K|^{\alpha}\left\{1-i \beta \operatorname{sing}(k) \tan \frac{\Pi \alpha}{2}\right\}, \\
i \delta k-\gamma|k|\left\{1+i \beta \operatorname{sing}(k) \frac{2}{\Pi}\right\}, \\
(\alpha=1),
\end{array}\right. \\
& \operatorname{sing}(k)=\frac{k}{|k|} .
\end{aligned}
$$

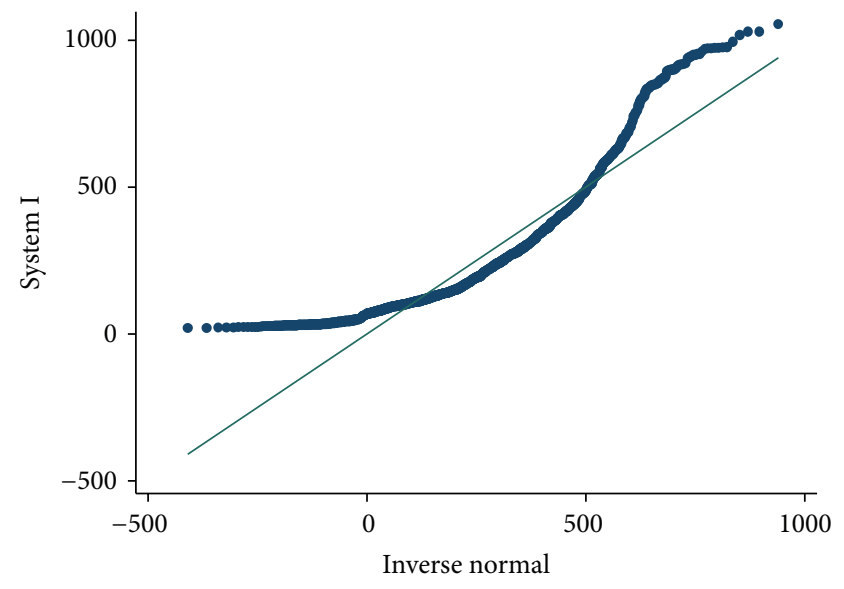

FIGURE 1: Quantile-quantile normal plot of IEE-104 bus system.

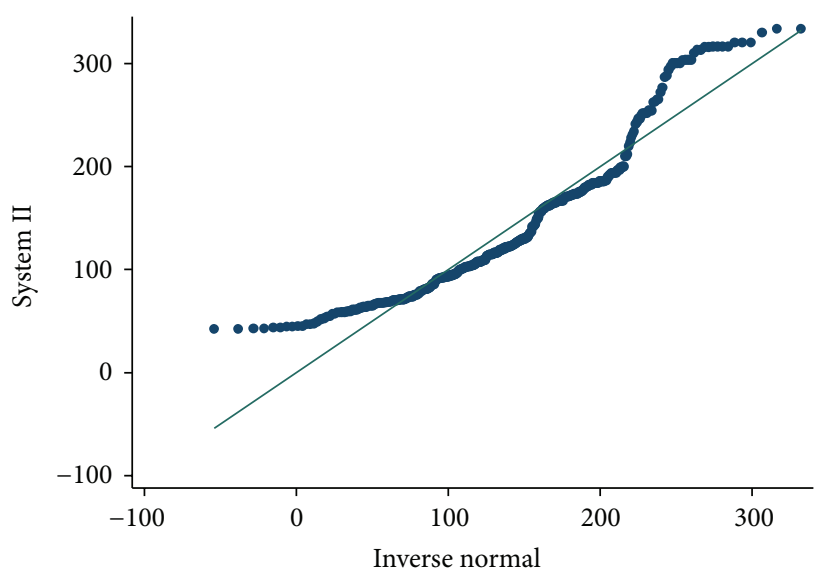

FIGURE 2: Quantile-quantile normal plot of IEEE-24 bus system.

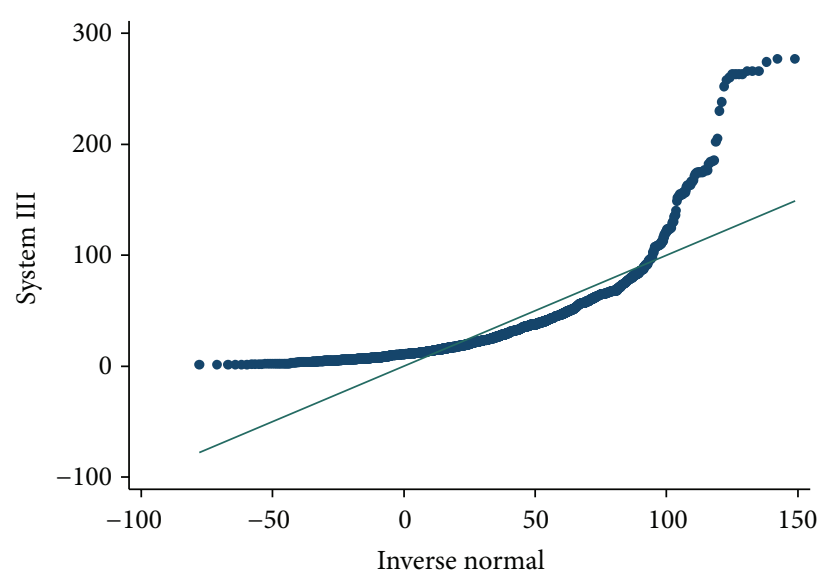

FIGURE 3: Quantile-quantile normal plot of IEEE-118 bus system.

Adjustments of the data were obtained parameters characterizing the distribution of the three methods used generally, maximum likelihood, quantile, and regression [13]. In this case, the regression method is used since it is the method generally used in the analysis of stable series that has a better adjustment of the tails of the distribution. 
TABLE 1: Shapiro-Wilk normal test.

\begin{tabular}{lccccc}
\hline Variable & Obs & $w$ & $v$ & $z$ & $P$ value $5 \%$ \\
\hline System I & 1541 & 0.86224 & 128.857 & 12.239 & 0.0000 \\
System II & 408 & 0.91322 & 24.319 & 7.600 & 0.0000 \\
System III & 2376 & 0.72011 & 388.189 & 15.259 & 0.0000 \\
\hline
\end{tabular}

TABLE 2: Alpha stable parameters with three methods of estimation.

\begin{tabular}{lcccc}
\hline & $\alpha$ & $\beta$ & $\gamma$ & $\delta$ \\
\hline $\begin{array}{l}\text { IEE-104 bus system } \\
\quad \text { Maximum likelihood }\end{array}$ & $\mathbf{1 . 2 0 0 0}$ & $\mathbf{1 . 0 0 0 0}$ & $\mathbf{8 4 . 3 9 5 4}$ & $\mathbf{4 2 3 . 4 1 4}$ \\
$\quad$ Quantile & 1.6132 & 1.0000 & 121.3540 & 291.3820 \\
$\quad$ Regression & 1.6938 & 1.0000 & 113.085 & 227.7650 \\
IEE-24 bus system & & & & \\
$\quad$ Maximum likelihood & 1.4000 & 1.0000 & 35.4172 & 160.6370 \\
$\quad$ Quantile & 1.6045 & 1.0000 & 41.7026 & 151.6880 \\
$\quad$ Regression & 1.8430 & 1.0000 & 42.8166 & 121.925 \\
$\quad$ IEE-118 bus system & & & & \\
$\quad \begin{array}{l}\text { Maximum likelihood } \\
\quad \text { Quantile }\end{array}$ & 1.2000 & 1.0000 & 10.7543 & 54.0993 \\
$\quad$ Regression & 1.5539 & 1.0000 & 15.1875 & 32.7828 \\
\hline
\end{tabular}

TABLE 3: Alpha stable parameters.

\begin{tabular}{lcccccc}
\hline System & \multirow{2}{*}{$\alpha$} & \multirow{2}{*}{$\beta$} & \multirow{2}{*}{$\gamma$} & $\delta$ & $\begin{array}{c}\text { Anderson } \\
\text { darling statistical }\end{array}$ & $\begin{array}{c}P \text { value } \\
5 \%\end{array}$ \\
\hline I & 1.6938 & 1.0000 & 113.085 & 227.765 & 0.623263 & 0.62625 \\
II & 1.8430 & 1.0000 & 42.8166 & 121.925 & 3.22121 & 0.02116 \\
III & 1.5092 & 1.0000 & 13.3914 & 35.9108 & 1.40198 & 0.20170 \\
\hline
\end{tabular}

The parameters for the alpha stable distribution show a parameter alpha different to two (normal case) that means the data has impulsivity. To test the fit of the distribution to data, many authors agree with the utility of Anderson-Darling [14] test for data with fat tails, and in all cases the null hypothesis was not rejected.

The parameter alpha in three systems shows the presence of impulsivity in the series, all series are symmetric positive and have high dispersion. That depends on the demand in the 24 hours of day and shows the habits of consumption. Figures 4, 5, and 6 show the fit of data wit alpha stable distribution through Quantile-Quantile stable plots. The tails have been captured and this information is considered into the simulation of random alpha stable variables.

The parameter alpha shows the impulsivity of data, to simulate the random alpha stable variables, was considered three scenarios with $\alpha=1.50, \alpha=1.69$, and $\alpha=1.84$. Other parameters used to simulate random alpha stable variables are the maximum of the parameters because that helps to simulate extreme values in dispersion and localization. In Figure 7 we can see the effect to the parameter alpha on the distribution function.

With the data the three scenarios are considered to simulate the random variables, with the next set of parameters $\alpha=(1.59,1.69,1.84), \beta=1.0, \gamma=(13.3,42.8,113.08)$, and

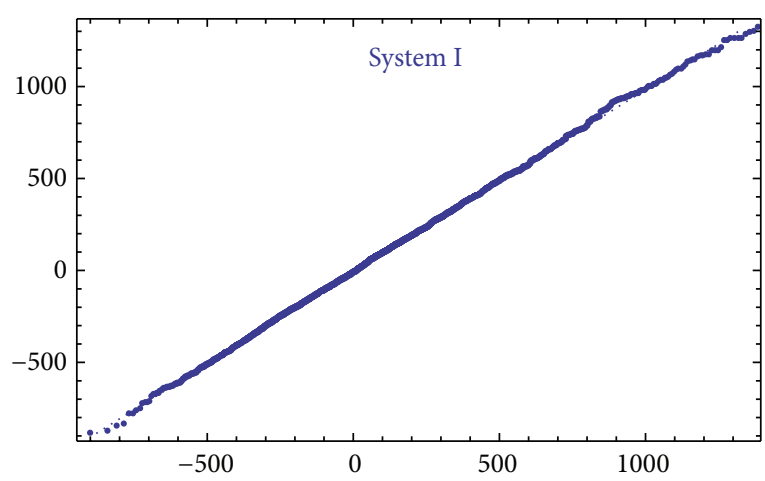

FIGURE 4: Quantile-quantile stable plots of 104-bus system.

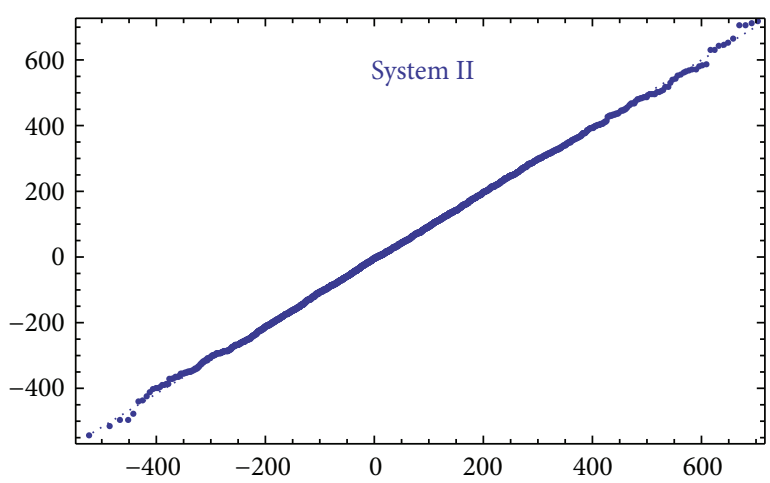

FIGURE 5: Quantile-quantile stable plots of IEEE-24 bus system.

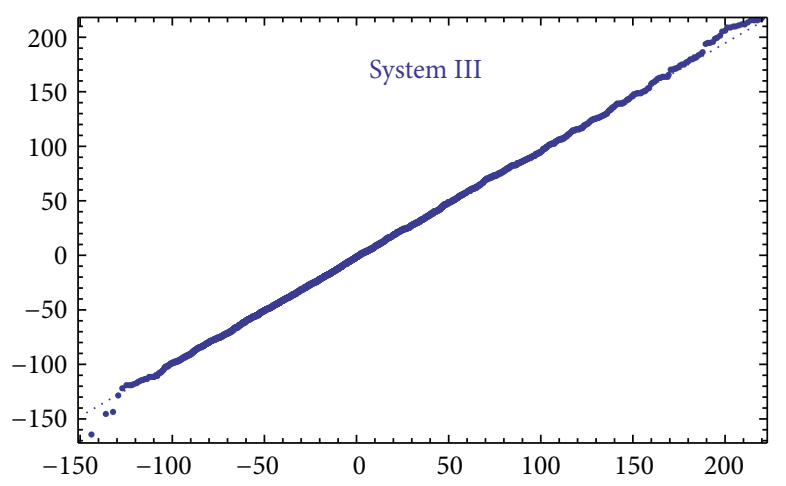

FIgURE 6: Quantile-Quantile stable plots of IEEE-118 bus system.

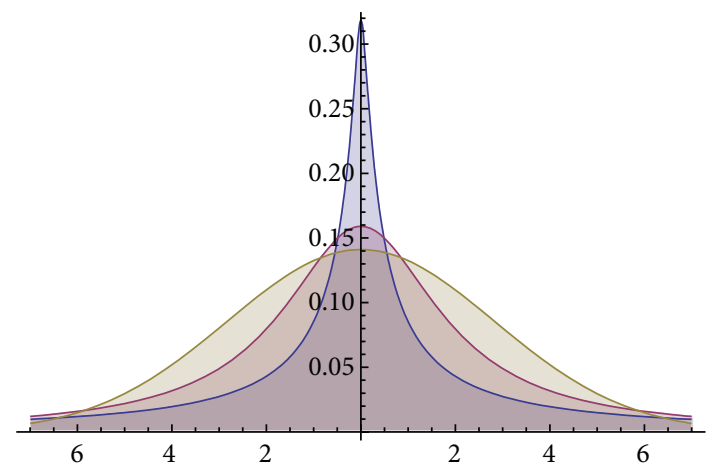

FIGURE 7: Stable distribution in three scenarios of alpha parameter $(\alpha=0.5,1$, and 2$)$. 


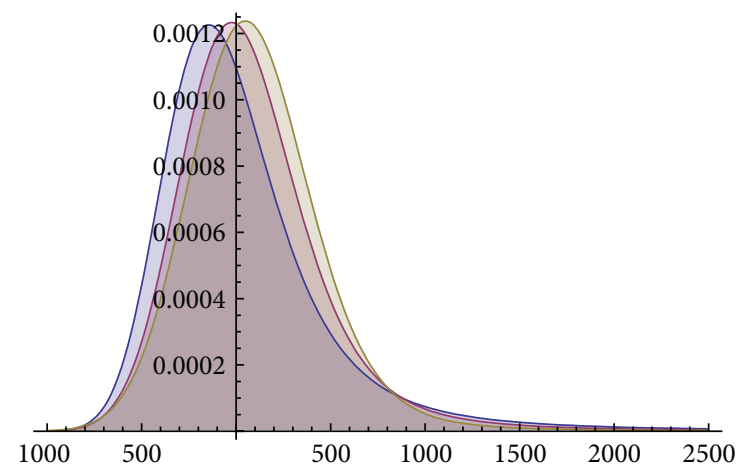

FIGURE 8: Stable distribution for simulations in three scenarios of alpha parameter $(\alpha=1.59,1.69$, and 1.84).

$\delta=(35.9,121.9,227.76)$. The effect of parameter alpha in the distribution function fitted is showed in Figure 8 . The parameter alpha measures the impulsivity of data that the reason because is one of the most important parameters for this analysis.

After the last analysis by Chambers-Mallows-Stuck method, we created 100 instances for each original test system according to the original energy demand ranges. The numerical behaviors of some of these instances are showed in next section.

\section{Computational Results}

In this section we present the results to the computational experiments that we carried out to evaluate the performance of the generated test systems by the proposed method. All simulations and mathematical models were carried out on an AMD Phenom II N970 Quad-Core with a $2.2 \mathrm{GHz}$ processor and 4 GB RAM. Table 4 shows the results of the Multiperiod Unit Commitment for 20 generated instances.

The mathematical model of Multiperiod Unit Commitment was implemented in GAMS [15] using the solver DICOPT [16] for solving the mixed integer nonlinear problems and CPLEX [17] for mixed integer problems.

Concerning the computational times, we note that they are more similar than original systems. One must highlight that the computational times do not exceed more than 35 minutes (See Figures 9, 10, and 11). In general, the generated instances do not have numerical problems in the optimization process, which normally occurs in Unit Commitment Problem. Only for a one instance from IEEE-24 bust test system no solution was found. This is due to a problem of numerical stability.

\section{Conclusions}

By introducing Chambers-Mallows-Stuck method, we presented a new way to generate electrical test systems for economic analysis. We show that the electrical demand is an alpha stable random variable, so we use it to generate several instances for the original test systems. The results show that electrical demand presents a greater degree of impulsivity due
TABLE 4: Results obtained by DICOPT solver.

\begin{tabular}{lcccccc}
\hline & \multicolumn{2}{c}{ SYS-104 } & \multicolumn{2}{c}{ IEEE-24 } & \multicolumn{2}{c}{ IEEE-118 } \\
Instance & $\begin{array}{c}\text { Bound } \\
\$\end{array}$ & $\begin{array}{c}\text { Time } \\
\text { min }\end{array}$ & $\begin{array}{c}\text { Bound } \\
\$\end{array}$ & $\begin{array}{c}\text { Time } \\
\text { min }\end{array}$ & $\begin{array}{c}\text { Bound } \\
\$\end{array}$ & $\begin{array}{c}\text { Time } \\
\text { min }\end{array}$ \\
\hline I1 & 67398.22 & 34 & 5616.41 & 18 & 1215.18 & 23 \\
I2 & 67281.37 & 34 & 5665.61 & 18 & 1237.5 & 23 \\
I3 & 67104.12 & 32 & 5669.13 & 18 & 1296.74 & 22 \\
I4 & 67623.65 & 34 & 5711.97 & 19 & 1307.85 & 30 \\
I5 & 67207.35 & 33 & 5579.78 & 19 & 1284.37 & 24 \\
I6 & 67027.43 & 35 & 5699.76 & 18 & 1286.55 & 28 \\
I7 & 67250.75 & 34 & 5603.32 & 19 & 1290 & 24 \\
I8 & 67211.65 & 33 & 5670.61 & 19 & 1327.28 & 26 \\
I9 & 67708.45 & 33 & 5543.96 & 18 & 1294.47 & 24 \\
I10 & 67088.38 & 34 & 5453.76 & 19 & 1354.32 & 22 \\
I11 & 68018.36 & 33 & 5484.37 & 19 & 1366.83 & 24 \\
I12 & 69096.21 & 33 & 5283.34 & 18 & 1379.43 & 23 \\
I13 & 68526.73 & 32 & 5408.32 & 17 & 1385.47 & 25 \\
I14 & 69827.1 & 33 & 5491.87 & 20 & 1392.12 & 25 \\
I15 & 70466.12 & 34 & 5192.34 & 20 & 1425.01 & 22 \\
I16 & 71917.87 & 32 & 5204 & 20 & - & - \\
I17 & 70696.73 & 34 & 5072 & 20 & 1275.4 & 23 \\
I18 & 71597.29 & 35 & 5562.65 & 18 & 1330.22 & 23 \\
I19 & 72048.48 & 33 & 5988.54 & 18 & 1348.93 & 23 \\
I20 & 70726.82 & 34 & 6202.27 & 19 & 1380.87 & 25 \\
\hline
\end{tabular}

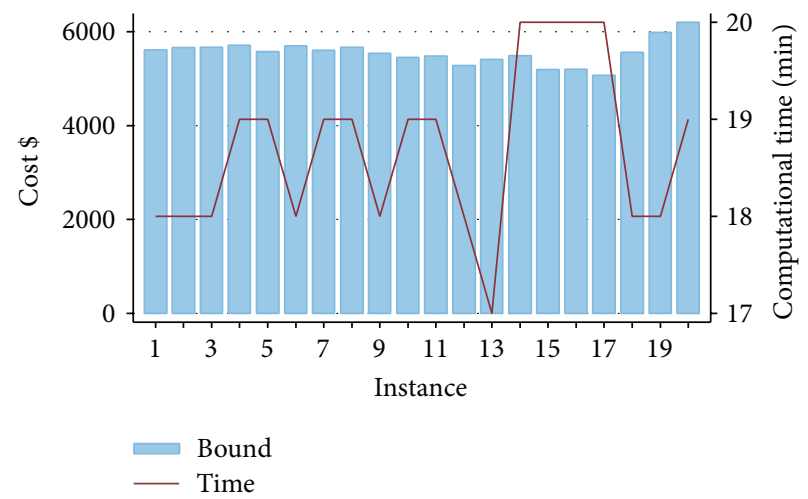

FIGURE 9: Bounds and computational time in IEEE-24 bus system.

to the presence of peaks in the series during the hours of the day and seasons of high energy demand.

We use Chambers-Mallows and Stuck algorithm for simulating alpha stable random variables characterizing demand patterns of real systems. By modeling the demand through the use of alpha stable distribution can catch the real behavior of the electrical demand and build possible extreme scenarios, each scenario corresponds to a price-elastic demand curve. The simulations were based on two IEEE test systems and a portion of energy system of Mainland Spain.

We test all generated instances in the Multiperiod Unit Commitment Problem and the results show that the proposed 


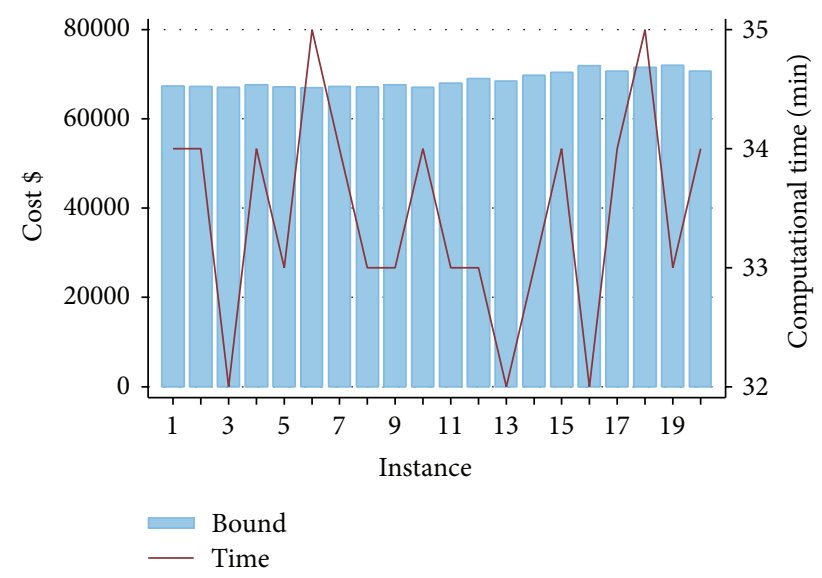

FIGURE 10: Bounds and computational time in Sys-104 bus system.

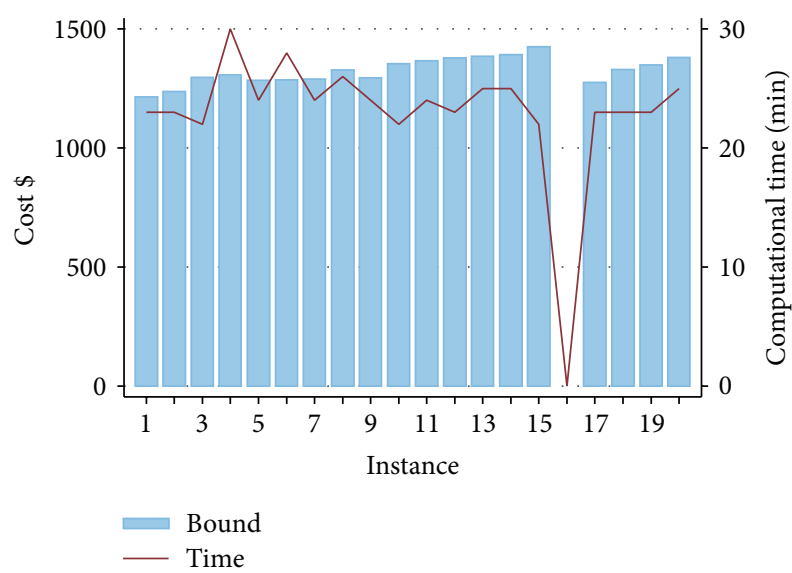

FIGURE 11: Bounds and computational time in IEEE-118 bus system.

methodology is relevant, obtaining feasible solutions with GAMS solver in the same way of the original systems.

This work contributes to having standardized test systems that can be used to benchmark the performance of many proposed techniques.

\section{Conflict of Interests}

The authors declare that there is no conflict of interests regarding the publication of this paper.

\section{References}

[1] A. J. Wood and B. F. Wollenberg, Power Generation, Operation and Control, John Wiley \& Sons, 1996.

[2] A. L. Diniz, "Test cases for unit commitment and hydrothermal scheduling problems," in Proceedings of the IEEE Power and Energy Society General Meeting (PES '10), pp. 1-8, IEEE, Minneapolis, Minn, USA, July 2010.

[3] X.-P. Zhang and C. S. Schaffner, "Minutes of the Working Group meeting on Test Systems for Economic Analysis, 2007, 2008, 2009 and 2010".
[4] P. Lévy, "Théorie des erreurs la loi de Gauss et les lois exceptionelles," Bulletin de la Société Mathématique de France, vol. 52, pp. 49-85, 1924.

[5] P. Charman, P. Bhavaraju, and R. Billington, "IEEE reliability test system," IEEE Transactions on Power Apparatus and Systems, vol. 98, no. 6, pp. 2047-2054, 1979.

[6] R. Christie, "IEEE 118 bus test case," College of Engineering, Electric Engineering, University of Washington, 1993, http:// www.ee.washington.edu/research/pstca.

[7] N. Alguacil and A. J. Conejo, "Multiperiod optimal power flow using benders decomposition," IEEE Transactions on Power Systems, vol. 15, no. 1, pp. 196-201, 2000.

[8] J. P. Nolan, Modeling Financial Data with Stable Distributions, Department of Mathematics and Statistics, American University, 2005, http://academic2.american.edu/ jpnolan/.

[9] I. Belov, A. Kabasinskas, and L. Sakalauskas, "A study of stable models of stock markets," Information Technology and Control, vol. 35, no. 1, pp. 34-56, 2006.

[10] J. M. Chambers, C. L. Mallows, and B. W. Stuck, "A method for simulating stable random variables," Journal of the American Statistical Association, vol. 71, no. 354, pp. 340-344, 1976.

[11] J. A. Marmolejo, I. Litvinchev, R. Aceves, and J. M. Ramirez, "Multiperiod optimal planning of thermal generation using cross decomposition," Journal of Computer and Systems Sciences International, vol. 50, no. 5, pp. 793-804, 2011.

[12] G. Samorodnitsky and M. S. Taqqu, Stable Non-Gaussian Random Processes: Stochastic Models with Infinite Variance, Chapman and Hall, New York, NY, USA, 1994.

[13] J. P. Nolan, Fitting Data and Assessing Goodness-of-Fit with Stable Distributions, Department of Mathematics and Statistics, American University, 1999, http://academic2.american.edu/ jpnolan/.

[14] M. A. Stephens, "Goodness of fit for the extreme value distributions," Biometrika, vol. 64, no. 3, pp. 583-588, 1977.

[15] A. Brooke, D. Kendrick, and A. Meeraus, GAMS: A User's Guide, The Scientific Press, 1998.

[16] A. Brooke, D. Kendrick, and A. Meeraus, GAMS/DICOPT. User Notes, GAMS Development Corporation, 2010.

[17] A. Brooke, D. Kendrick, and A. Meeraus, GAMS/Cplex 12. User Notes, GAMS Development Corporation, 2010. 


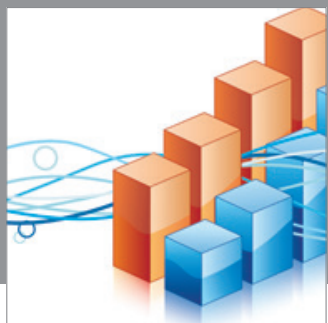

Advances in

Operations Research

mansans

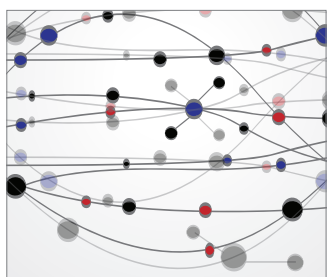

The Scientific World Journal
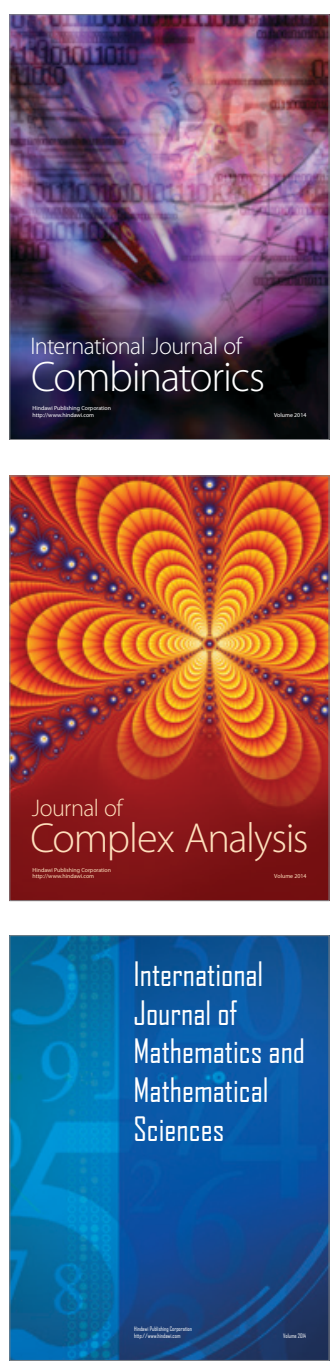
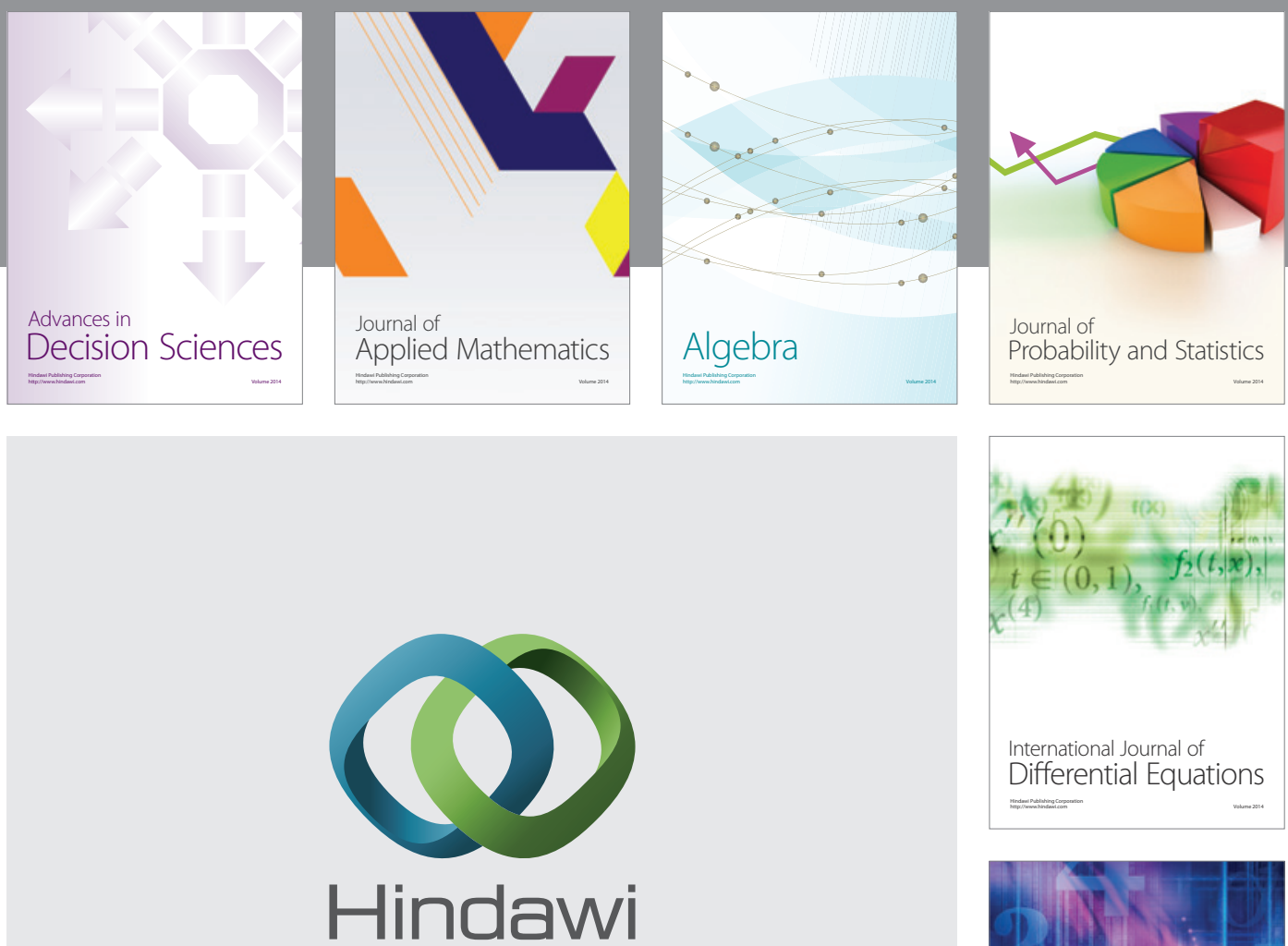

Submit your manuscripts at http://www.hindawi.com
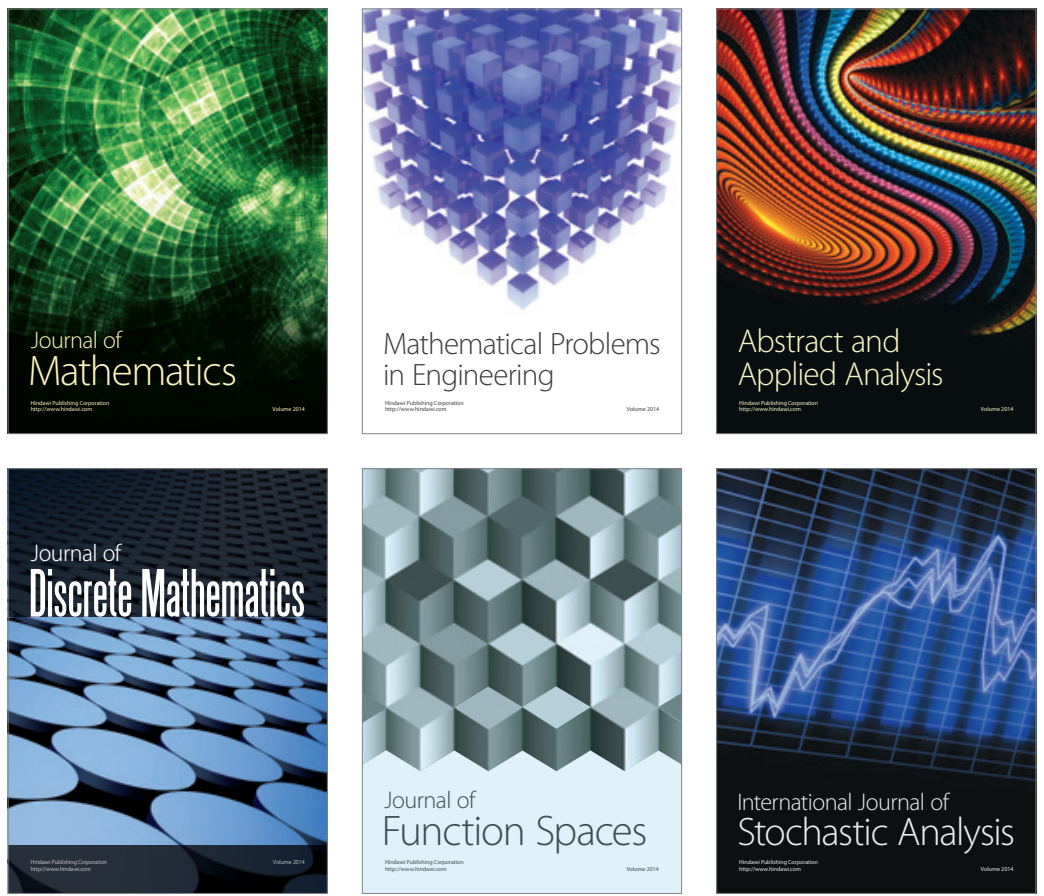

Journal of

Function Spaces

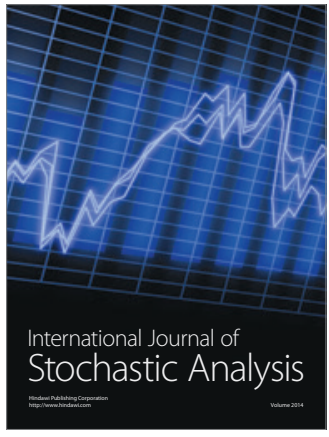

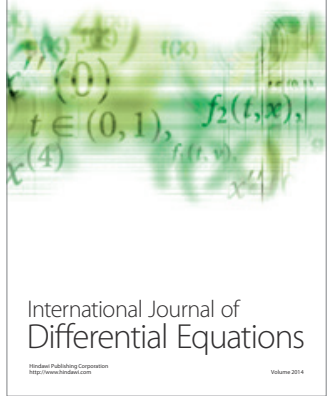
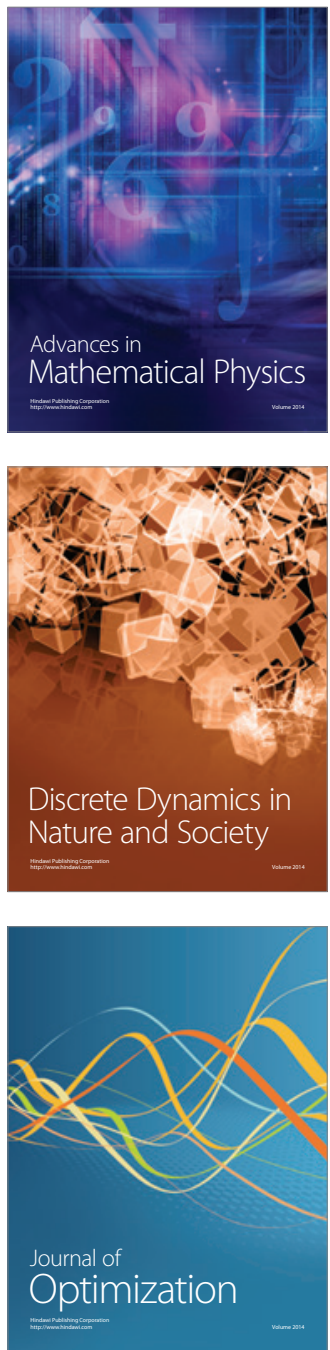